\title{
Different Redox Response Elicited by Naturally Occurring Antioxidants in Human Endothelial Cells
}

\author{
Roberta Giordo ${ }^{1}$, Annalisa Cossuํㄹ ${ }^{1}$ Valeria Pasciu ${ }^{4}$, Phu Thi Hoa ${ }^{1,5}$, Anna Maria Posadino ${ }^{1}$ and \\ Gianfranco Pintus ${ }^{1,2,3, *}$ \\ ${ }^{1}$ Laboratory of Vascular Biology, Department of Biomedical Sciences, University of Sassari, Sassari, Italy \\ ${ }^{2}$ Centre of Excellence for Biotechnology Development and Biodiversity Research, University of Sassari, Sassari, Italy \\ ${ }^{3}$ National Institute of Biostrucures and Biosystems, University of Sassari, Sassari, Italy \\ ${ }^{4}$ Department of Veterinary Medicine, University of Sassari, Sassari, Italy \\ ${ }^{5}$ Department of Biochemistry, Huè University of Medicine and Pharmacy, Huè, Vietnam
}

\begin{abstract}
Evidences that higher natural antioxidant (NA) intake provides protection against cardiovascular disease (CVD) are contradictory. Oxidative-induced endothelial cells (ECs) injury is the key step in the onset and progression of CVD and for this reason the cellular responses resulting from NA interaction with ECs are actively investigated. This study was designed to investigate the direct impact of different naturally occurring antioxidants on the intracellular ROS levels in cultured human ECs. NA-induced redox changes, in terms of modulation of the intracellular ROS levels, were assessed by using the ROS fluorescent probe 2',7'-dichlorodihydrofluorescein diacetate $\left(\mathrm{H}_{2} \mathrm{DCF}-\mathrm{DA}\right)$. While caffeic and caftaric acid exerted an anti-oxidant effect, both coumaric acid and resveratrol were pro-oxidant. Anti- and pro-oxidant effects of the tested compounds were concentration dependent, showing the induction or the tendency to promote a prooxidant outcome with increasing concentrations. Interestingly, the anti- and pro-oxidant behavior of chlorogenic and ferulic acid was dependent on the basal intracellular redox state. Our data indicate that naturally occurring antioxidants are able to induce a rapid modification of the intracellular ROS levels in human ECs, which is dependent on both the applied concentration and the intracellular redox state.
\end{abstract}

Keywords: ROS, natural phenolic antioxidants, endothelial cells, anti- and pro-oxidant effect.

\section{INTRODUCTION}

Reactive oxygen species (ROS) have been traditionally regarded as toxic products of metabolism because of their role in affecting cellular physiology by damaging structural and functional molecules such as lipids, proteins and DNA [1]. The increasing common opinion that the consumption of antioxidant molecules is useful to decrease ROS concentration and that this may promote good health by preventing or alleviating diseases, is greatly increasing the uptake of foods containing antioxidants. Naturally occurring antioxidants (NA) from vegetable foods and some beverages such as tea or coffee and red wine, have been associated with a reduced incidence of risk factors for a number of cardiovascular diseases (CVD), including atherosclerosis and hypertension. Evidences supporting this hypothesis are based on epidemiological observations, animal studies and cell culture experiments [2]. In particular, food-derived NA, as a phenolic or polyphenolic compounds, have been proven to be effective in counteracting endothelial cell (EC) activation by inhibiting the expression of adhesion molecules such as

*Address correspondence to this author at the Department of Biomedical Sciences, Viale San Pietro 43/B, 07100 Sassari, Italy;

Tel.: +39 079228 121; Fax: +39 079228 120; E-mail: gpintus@uniss.it vascular cell adhesion molecule-1 (VCAM-1), intercellular adhesion molecule 1 (ICAM-1) and E-selectin [3], thereby lowering cellular atherogenic properties and inhibiting vascular damage. The vascular protective effect ascribed to NA seems due, at least in part, to their ability to prevent cellular oxidative damage as a result of their well-documented ROS scavenger activity $[4,5]$. Indeed, ROS released endogenously by vascular cells in response to pro-atherosclerotic factors, can prompt vascular damage and arterial remodeling by targeting several intracellular pathways [6]. Downstream ROS-activated signaling mechanisms can affect EC biochemical and cellular behavior such as apoptosis and proliferation, leading to endothelial dysfunction, an initial episode progressing toward atherosclerosis and hypertension [7, 8]. Nonetheless, many controlled clinical trials have failed to show a consistent benefit of natural antioxidants on several CVD and its sequelae [9]. The reason for the disappointing findings is unclear, but some possible explanations have been proposed. Compelling evidence suggests that in the endothelium, ROS are not only harmful compounds but also essential participants in cellular signalling [10]. ROS such as superoxide anions and hydrogen peroxide appear to be transiently produced in response to growth factor and cytokine stimulation. This ligand-stimulated change in the cellular re- 
dox state participates in downstream signal transduction by modulating activity of enzymes and transcriptions factors, which are crucial regulators of EC proliferation and angiogenesis [10]. Thus, endothelial production of ROS appears both to sustain growth and to lead to cellular death, and ROS, by simultaneous functioning as pro- and anti-apoptotic messengers, have both a protective and deleterious role in EC [7]. In addition, natural antioxidants, do not always behave like antioxidants; depending upon the concentration $[11,12]$ and environmental oxidative status [13, 14], they can in fact have a pro-oxidant effect thereby causing reversible or irreversible cellular damage. In this light, antioxidantbased strategies aimed at inhibiting the production and/or action of intracellular ROS, with the goal of preventing endothelial damage or dysfunction, may have the untoward effect of leading to EC loss and therefore promote CVD. Despite the large number of papers reporting the protective effect exerted by natural antioxidants against diseases or toxic drugs, there is a paucity of research on their possible toxicity. In particular, relatively few reports analyzed the effect of naturally occurring antioxidants on the intracellular ROS levels in unstressed human EC.

Hence, the present work was undertaken with the intent to investigate the effect of different NA on the intracellular ROS levels of human ECs. Here we report that different food-contained NA are able to rapidly modify the intracellular ROS levels in cultured human EC. The anti- and prooxidant effects of the tested compounds depend upon the concentrations used and the basal intracellular ROS level. We also provide evidence that antioxidant-induced increase of the intracellular ROS levels is mediated by flavincontaining oxidases.

\section{METHODS}

\subsection{Reagents}

Antioxidants: caffeic acid, coumaric acid, chlorogenic acid, caftaric acid and ferulic acid were prepared by the Istituto Agrario sperimentale San Michele all'Adige (Trento, Italy), resveratrol was supplied by Sigma (St. Louis, MO). The concentrations used for all the antioxidants were in a range between 0.5-50 $\mu \mathrm{M}$. Indeed, several published works show that NA such as Resveratrol for instance, can reach in vivo concentrations comparable to the ones we have chosen for this experimental study. Each freeze-dried antioxidant was dissolved in ethanol solvent and stored at $-20^{\circ} \mathrm{C}$. Inhibitors of ROS-generating enzymes used where: Rotenone (10 $\mu \mathrm{M})$, 4-(2-aminoethyl) benzenesulfonyl fluoride (AEBSF) $(10 \mu \mathrm{M})$ both from Sigma-Aldrich (St. Louis, MO); N (G)nitro-L- arginine methyl ester (L-NAME) (1 mM), diphenylene iodonium (DPI) $(20 \mu \mathrm{M})$ and apocynin $(30 \mu \mathrm{M})$ all from Calbiochem (EMD Chemicals Inc San Diego, CA).

\subsection{Cell Culture and Treatments}

Primary human ECs were isolated from human umbilical cords and cultured as previously described $[15,16]$. Briefly, ECs were detached from the interior of the umbilical vein of a $30 \mathrm{~cm}$ segment cord by treatment for $10 \mathrm{~min}$ at $37^{\circ} \mathrm{C}$ with $0.05 \%(\mathrm{w} / \mathrm{v})$ collagenase type II from Clostridium hystolyticum (Sigma) in medium M199 (Invitrogen) containing 100
$\mathrm{U} / \mathrm{ml}$ of penicillin $\mathrm{G}$ sodium salt and $100 \mu \mathrm{g} / \mathrm{ml}$ streptomycin sulfate (Sigma). ECs were harvested by centrifugation at $1000 \times \mathrm{g}$ for $10 \mathrm{~min}$ and resuspended in $5 \mathrm{ml}$ medium M199 supplemented with $10 \%(\mathrm{v} / \mathrm{v})$ foetal calf serum (FCS), $10 \%$ (v/v) newborn-calf serum (Invitrogen, Carlsbad, CA), $2 \mathrm{mM}$ glutamine and antibiotics. Cells were then plated in $25 \mathrm{~cm}^{2}$ tissue culture flasks (Falcon, Oxnard, CA) pre-treated with $0.1 \%$ gelatin and cultured in an atmosphere of $5 \% \mathrm{CO}_{2} / 95 \%$ air. Cultured cells were identified as endothelial by their typical cobblestone appearance and production of Von Willebrand factor. ECs were used within three passages, and all the experiments were performed in 96-well black plates (Corning). In order to mimic physiological vessel wall conditions, before experimentation cells were grown until confluence to reach contact-dependent growth inhibition.

The levels of intracellular ROS were monitored in cultured ECs during a time course of 160 minutes under three different experimental conditions: i) single antioxi-dants; ii) single antioxidants plus selected ROS-generating enzymes inhibitors; iii) only ROS-generating enzymes inhibitors. Sites of intracellular ROS production were investigated by using different inhibitors of ROS-generating enzymes. Respiratory chain-deriving ROS generation was investigated using the complex I (NADH dehydrogenase) inhibitor rotenone $(10 \mu \mathrm{M})$ [17]. To study the contribution to ROS production of nicotinamide adenine dinucleotide phosphate-oxidase (NADPH)-dependent oxidases, we ran sets of experiments in the presence of the NADPH oxidase inhibitors 4-(2-Aminoethyl) benzenesulfonyl fluoride hydrochloride (AEBSF) (10 $\mu \mathrm{M})$ and apocynin $(30 \mu \mathrm{M})$ [18], while the broad involvement of flavoprotein oxidases was investigated by using diphenylene iodonium (DPI) $(20 \mu \mathrm{M})$, which is a general flavin-containing enzymes inhibitor [19]. L-NG-Nitroarginine methyl ester (L-NAME) (1 mM), a nitric oxide synthase (NOS) inhibitor, was used to test NOS involvement in ROS production [20].

\subsection{Measurements of Intracellular ROS Levels}

Intracellular ROS levels were determined by using the ROS molecular probe 2',7'-dichlorodihydrofluorescein diacetate $\left(\mathrm{H}_{2} \mathrm{DCF}-\mathrm{DA}\right)$ (Invitrogen) [21]. Within the cell, esterases cleave the acetate groups on $\mathrm{H}_{2}$ DCF-DA, thus trapping the reduced form of the probe $\left(\mathrm{H}_{2} \mathrm{DCF}\right)$. Intracellular ROS oxidize $\mathrm{H}_{2} \mathrm{DCF}$, yielding the fluorescent product, DCF. For ROS measurements cultured cells were pre-incubated for 30 minutes with $200 \mu \mathrm{l}$ of PBS plus $(120 \mathrm{mM} \mathrm{NaCl}, 2.5 \mathrm{mM}$ $\mathrm{KCl}, 8.5 \mathrm{mM} \mathrm{NaH} \mathrm{PO}_{4}, 1.5 \mathrm{mM} \mathrm{KH}_{2} \mathrm{PO}_{4}, \mathrm{CaCl}_{2} 0.5 \mathrm{mM}$, $\mathrm{MgCl}_{2} 1 \mathrm{mM}, \mathrm{C}_{6} \mathrm{H}_{12} \mathrm{O}_{6} 30 \mathrm{mM}$ ) containing $3 \mu \mathrm{M} \mathrm{H}_{2}$ DCFDA, then washed with PBS and treated as described. Fluorescence was measured by using a Tecan GENios plus microplate reader (Tecan, Männedorf, Switzerland) in a lightprotected condition. Excitation and emission wavelengths used for fluorescence quantification were $485 \mathrm{~nm}$ and 535 $\mathrm{nm}$ respectively. Treatments-induced variation of fluorescence was kinetically measured every minute for 120 consecutive minutes. All fluorescence measurements were corrected for background fluorescence and protein concentration. Using untreated cells as a reference the anti- and prooxidant outcome was evaluated by comparison of five measurements and expressed as a percentage of untreated controls. 


\subsection{Statistical Analysis}

Data are expressed as means \pm S.D. of at least five different experiments. One-way analysis of variance followed by a post-hoc Newman-Keuls Multiple Comparison Test were used to detect differences of means among treatments with significance defined as $\mathrm{P}<0.05$ (GraphPad Prism version 5.00 .

\section{RESULTS}

\subsection{Effect of Natural Antioxidants on Intracellular ROS Levels in Human Endothelial Cells}

Here we assessed the effect of different natural antioxidants on rapid intracellular ROS modification in cultured human ECs. Naturally occurring phenolic compounds, such as resveratrol, coumaric acid, caffeic acid and chlorogenic acid (Fig. 1), have been extensively employed as antioxidant because of their ability to counteract oxidative stress. However, data concerning their direct anti- and pro-oxidant effect, in terms of ability to rapidly modulate the levels of intracellular ROS in unstressed normal human ECs are lacking. To investigate such aspects, cells were incubated for $30 \mathrm{~min}-$ utes with $1 \mu \mathrm{M} \mathrm{H}_{2}$ DCF-DA and then treated with the indicated NA. Under these experimental conditions the levels of intracellular ROS were kinetically assessed during a timecourse of 120 minutes. Using as reference untreated cells, the overall cellular redox response was evaluated by comparison of the slope obtained from each single kinetic fluorescence measurement ( $\mathrm{n}=5$ or more). Exposition of ECs to $25 \mu \mathrm{M}$ of different antioxidants resulted in a diverse effect upon intracellular ROS generation. As shown in Fig. (2A), the addition of caffeic acid caused a decrease of the intracellular ROS production eliciting a significant anti-oxidant effect compared to untreated cells (inset Fig. 2A). On the contrary, the<smiles>Oc1ccc(/C=C/c2cc(O)cc(O)c2)cc1</smiles><smiles>O=C(/C=C/c1ccc(O)c(O)c1)OC1CC2(C(=O)O)CC(O)C(O)C1C2O</smiles><smiles>O=C(O)/C=C/c1ccc(O)c(O)c1</smiles>

addition of both coumaric acid and resveratrol promoted a rapid increase in the levels of intracellular ROS resulting in a significant pro-oxidant effect (inset Fig. 2A). Chlorogenic acid, after causing an initial drop of ECs ROS production, at later times of incubation (around $55 \mathrm{~min}$ ) aroused a raise in the levels of intracellular ROS exerting, even at less extent of coumaric acid and resveratrol, an overall pro-oxidant effect (inset Fig. 2A). As reported in Fig. (2B) and its inset, caftaric acid and ferulic acid showed an analogous behavior to thatevoked by chlorogenic acid and caffeic acid, respectively , probably because of the same chemical structure of the analogous antioxidants (Fig. 1).

\subsection{Anti- and Pro-oxidant Effect of Chlorogenic and Fer- ulic Acid Depends on Basal Intracellular Redox Condi- tions}

It is now becoming evident that some antioxidant compounds, depending upon the environment redox conditions, can have both anti and pro-oxidant behavior [13, 14]. In order to investigate such aspects in human ECs we ran sets of experiments assessing the antioxidant-induced redox modification in response to different initial intracellular ROS levels. Fig. (3, A-D) shows plots of the slope value (kinetic measurements of intracellular ROS levels after NA stimulation) expressed as \% vs the initial measured intracellular ROS level (first 5 minutes of basal ROS measurement before the stimulation with NA) for each tested antioxidants. As compared to untreated cells and regardless the initial measured RFU, coumaric acid and resveratrol $(25 \mu \mathrm{M})$ always elicited a pro-oxidant effect when applied for 120 minutes to ECs (Fig. 3 C and D). Fig. (3A) reports the behavior of caffeic acid in response to different initial redox condition, which turned out to be anti-oxidant at all times. Treatment of ECs with $25 \mu \mathrm{M}$ chlorogenic acid results in a dual effect,<smiles>COc1cc(/C=C/C(=O)O)ccc1O</smiles><smiles>O=C(C=Cc1ccc(O)c(O)c1)O[C@@H](C(=O)O)C(=O)OC(=O)C(Cl)(Cl)Cl</smiles>

Fig. (1). Name and chemical structure of the tested natural antioxidants. 
which was anti-oxidant at high initial ROS levels (above 18000 RFU) and pro-oxidant at low (Fig. 3B). Results similar to those reported for chlorogenic acid were also found in experiments performed with ferulic acid (Supplementary Fig. 1)

\subsection{Different Effect of Antioxidant Concentration on Endothelial Cell ROS Generation}

In order to investigate whether concentration could influence antioxidant-induced effect upon intracellular ROS levels, we ran sets of experiments in the presence of different doses of the tested compounds. Fig. (4A) and inset, clearly show that exposure of ECs to increasing concentrations of caffeic acid resulted in a dose-dependent raise of the intracellular ROS levels and a paralleled abrogation of its anti-oxidant effect. Thus caffeic acid acts as antioxidant at low doses,while it seems shifting toward a pro-oxidant behavior at increasing concentrations. Even though in a different concentration range, results analogous to Fig. (4A) were found when ECs were treated with the indicated concentrations of chlorogenic acid and resveratrol, Figs (4B and 4D) respectively. While at a concentration of $5 \mu \mathrm{M}$ chlorogenic acid showed an antioxidant effect, its outcome became significantly pro-oxidant at concentrations of $25 \mu \mathrm{M}$ and $50 \mu \mathrm{M}$ (Fig. (4B) and inset). Similarly, treatment of ECs with $0,5 \mu \mathrm{M}$ resveratrol resulted in a significant anti-oxidant effect, which shifted to prooxidant as a consequence of the increase of concentration (Fig. (4D) and inset). In the search for a similar result that we found for caffeic acid, resveratrol and chlorogenic acid, a larger number of doses were tested for coumaric acid. Starting at concentrations as low as $0.5 \mu \mathrm{M}$, the treatment of ECs with the indicated concentrations of coumaric acid dosedependently raised both intracellular ROS generation and
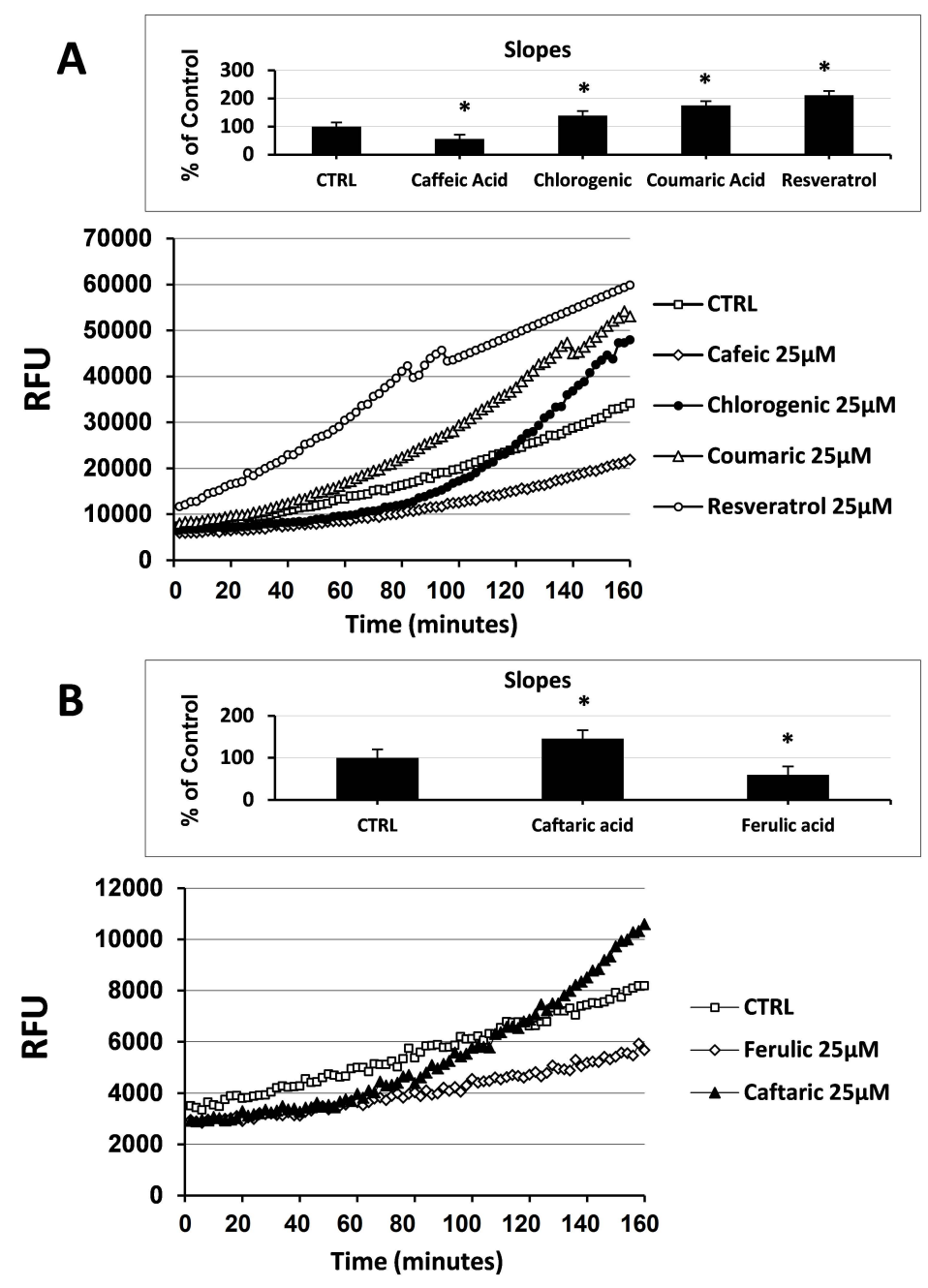

Fig. (2). Effect of natural antioxidants on intracellular ROS levels in cultured endothelial cells. (A) Measurement of Intracellular ROS generation in cultured human ECs in the absence (CTRL) or presence of $25 \mu \mathrm{M}$ caffeic acid, coumaric acid, chlorogenic acid and resveratrol. (B) Measurement of Intracellular ROS generation in cultured human ECs in the absence (CTRL) or presence of $25 \mu \mathrm{M}$ caftaric acid and ferulic acid. Treatment-induced variation of fluorescence was kinetically measured every minute for 120 consecutive minutes as described in materials and methods. (Figure insets): Using as reference untreated cells, the overall anti- and pro-oxidant effect was extrapolate by comparison of the slope obtained from at least five kinetic measurements for each experimental condition. Data are expressed as percentage of control and represent the means \pm SE of five different experiments. * significantly different from CTRL at $\mathrm{P}<0.05$. 

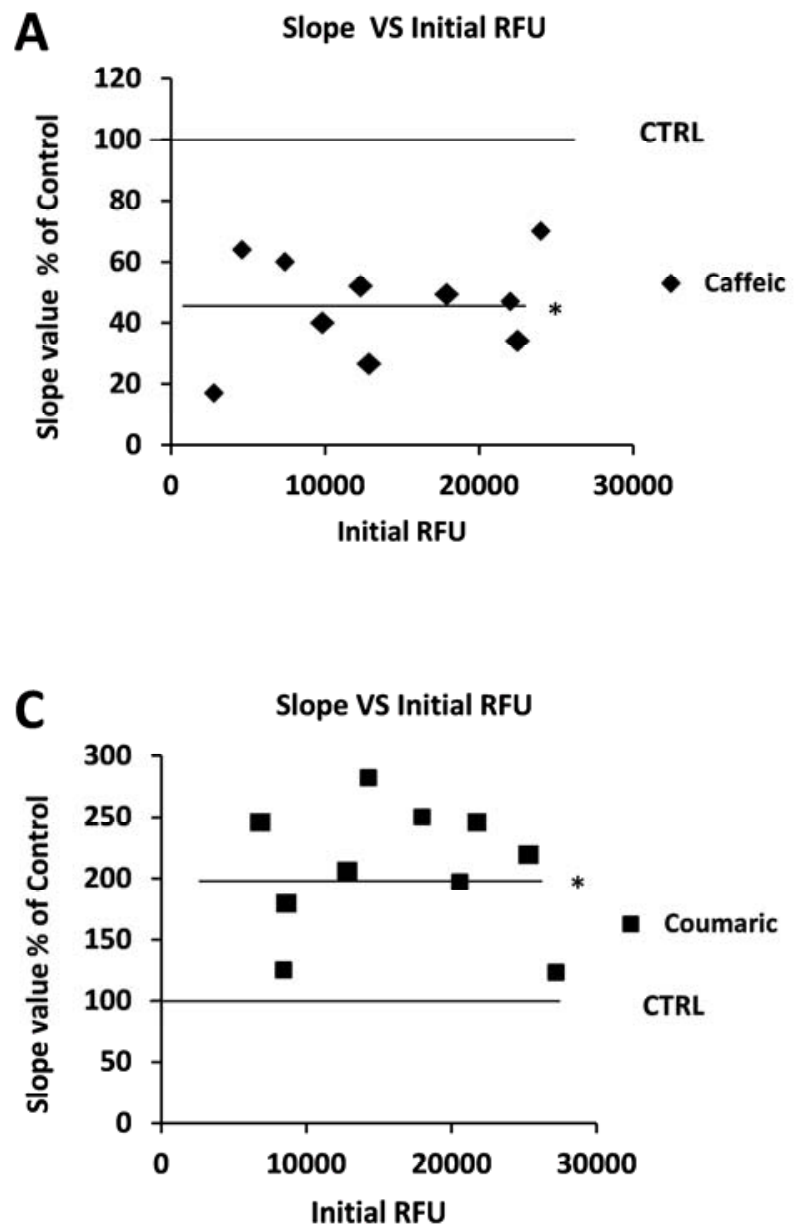
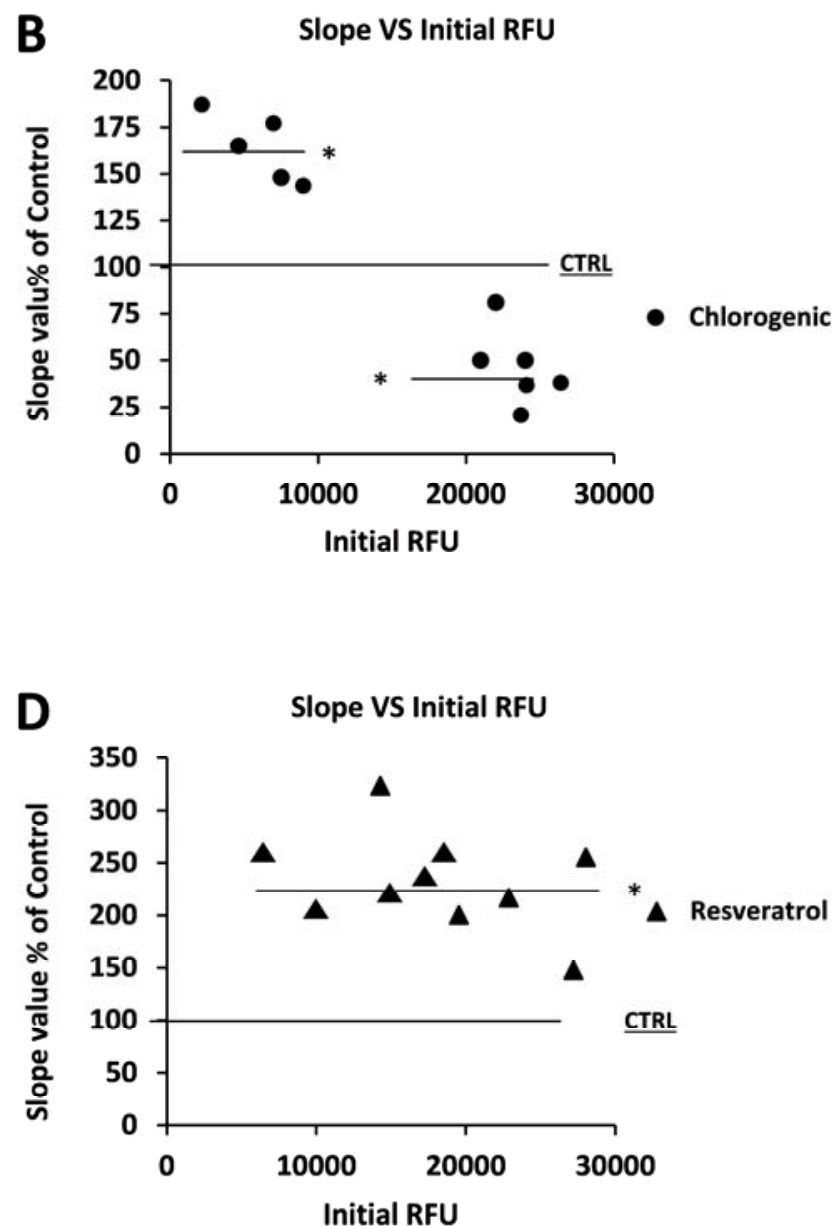

Fig. (3). Effect of the intracellular redox state on antioxidant-elicited anti- and pro-oxidant behaviour. Figure panels shows plots of the slope value expressed as \% vs the initial measured intracellular ROS level for (A) caffeic acid, (B) chlorogenic acid, (C) coumaric acid, (D) resveratrol. In panels A-D, the value of 100 in the y-axis refer to the slope value for a ROS kinetic measurements of untreated cultured human ECs (CTRL). Other data in the y-axis refer to the slope value obtained from five different ROS kinetic measurements of cultured human ECs treated for 120 minutes in the presence of $25 \mu \mathrm{M}$ of (A) caffeic acid, (B) chlorogenic acid, (C) coumaric acid, (D) resveratrol. Initial RFU value in the $\mathrm{x}$-axis for each panel refer to intracellular ROS measurements performed at the beginning of each experiments. In panels A-D, value in the y-axis that lie above 100 are considered as an antioxidant-induced pro-oxidant outcome, while value that lie below 100 are considered as an antioxidant-induced anti-oxidant outcome. Data in the $y$-axis are expressed as percentage of CTRL (untreated cells value=100).

* significantly different from CTRL at $\mathrm{P}<0.05$.

pro-oxidant effects, however the observed result was significantly different from the control beyond the dose of $12,5 \mu \mathrm{M}$ (Fig. (4C) and inset). At the lowest concentrations used (0,5 $\mu \mathrm{M})$, the treatment of EC with coumaric acid elicited a slight decrement of the intracellular ROS levels, which resulted in a reversal of its pro-oxidant effect. The current results clearly indicate a relationship between the dose applied and the potential ability of these molecules to act both as anti- and prooxidant.

\subsection{Effect of Different Inhibitors of ROS-generating En- zymes on Antioxidant-induced Intracellular ROS Levels Modification}

As reported in Fig. (3), at a concentration of $25 \mu \mathrm{M}$ and independently of the initial intracellular ROS levels, among all the tested antioxidants only coumaric acid and resveratrol were able to always exert a significant pro-oxidant effect. We therefore decided to investigate the potential intracellular source or ROS production generated in response to the aforementioned compounds. For this purpose, we made a set of experiments employing different inhibitors of some ROSgenerating enzymes. As shown in Fig. (5A), the presence of the complex I NADH dehydrogenase inhibitor rotenone failed to affect intracellular ROS generation both in control and coumaric acid-treated cells. Similar results were obtained when ECs were exposed to coumaric acid in the presence of the NOS inhibitor L-NAME and the NADPH oxidase Inhibitors AEBSF (Figs 5B and $\mathbf{C}$ ) and apocynin (not show). In agreement with the failure to affect intracellular ROS generation are the results displaying no significant modification in the overall anti- and pro-oxidant activity in ECs exposed to the tested ROS-generating enzymes inhibitors (inset Figs 5A-C). Instead, the general flavin-oxidase inhibitor DPI decreased both the coumaric acid-induced rise of intracellular ROS generation and pro-oxidant effects (Fig. (5D) and inset), clearly indicating the involvement of DPIsensitive flavin-containing oxidases (presumably endoplasmatic reticulum (ER)-resident oxidases) in the observed phe- 

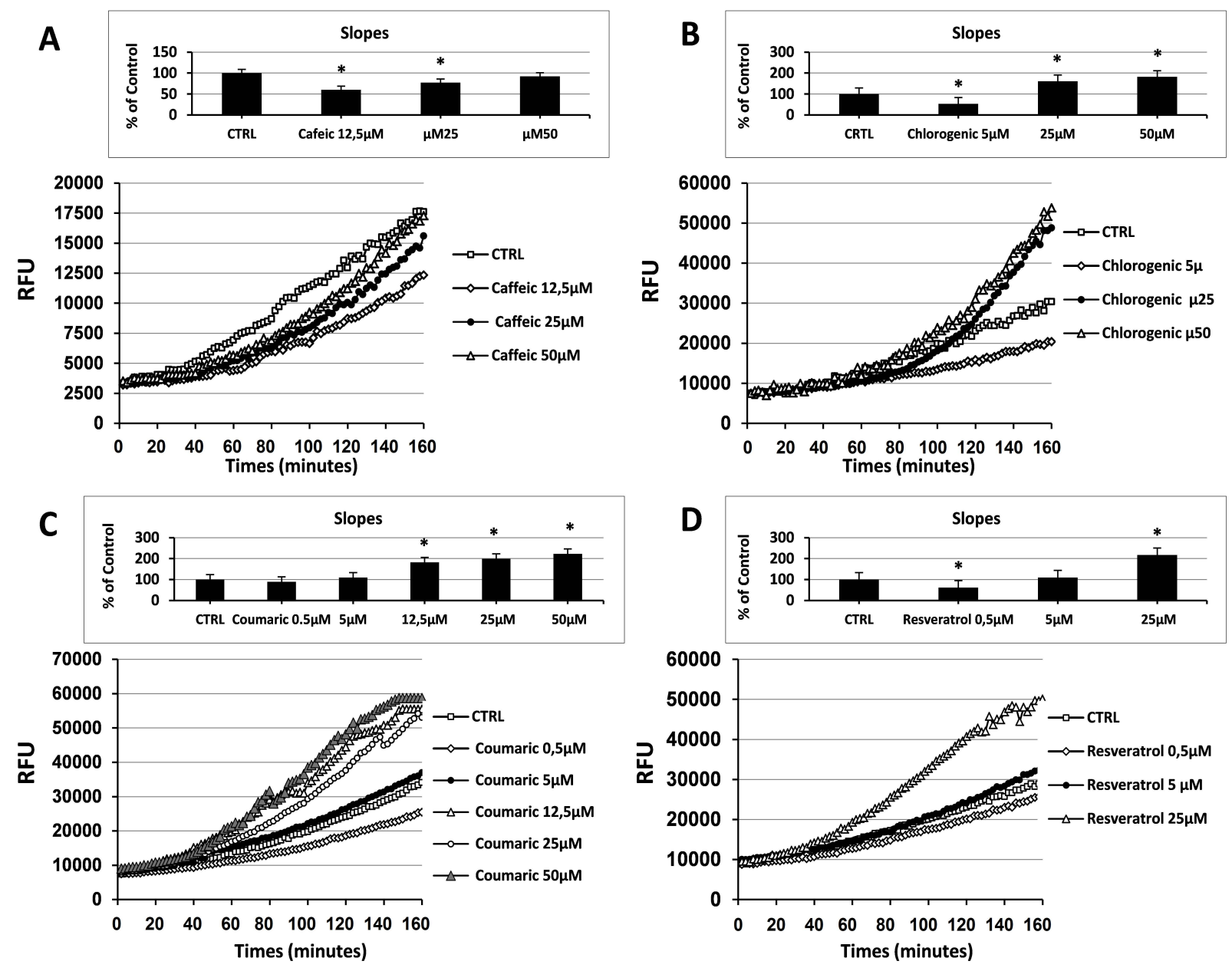

Fig. (4). Effect of natural antioxidants concentration on intracellular ROS levels in cultured endothelial cells. Measurement of Intracellular ROS generation in cultured human ECs in the absence (CTRL all panels) or presence of the indicated concentration of (A) caffeic acid, (B) chlorogenic acid, (C) coumaric acid, (D) resveratrol. Treatment-induced variation of fluorescence was kinetically measured every minute for 120 consecutive minutes as described in materials and methods. (Figure insets): Using as reference untreated cells, the overall pro-oxidant effect was extrapolate by comparison of the slope obtained from at least five kinetic measurements for each experimental condition. Data are expressed as percentage of control and represent the means \pm SE of five different experiments. * significantly different from CTRL.

nomena. Fig. (6A-D) show that the exposure of resveratroltreated cells to the aforementioned ROS-generating enzymes inhibitors resulted in analogous effects to those just presented for coumaric acid.

\section{DISCUSSION}

Evidence that higher NA intake from fruits and vegetables is associated with decreased risk for CVD is contradictory [22]. Food-derived phenolic compounds have powerful antioxidant effects in vitro in many test systems but can act as pro-oxidants in some others, therefore whether prooxidant or anti-oxidant effects potentially exerted by these compounds contribute to cardiovascular protection is uncertain. In the present work we report novel findings concerning the effect of relatively high-dose natural antioxidants on intracellular ROS modification in cultured human ECs. Our results provide evidence that molecules such as naturally occurring plant- and food-contained phenolic compounds (Fig.
1), which are widely regarded as active antioxidant and healthy compounds, depending upon the concentration and the cellular oxidative state, can deeply modify intracellular ROS levels eliciting a rapid redox change. In a set of experiments made with an explorative dose of $25 \mu \mathrm{M}$, the tested antioxidants exerted different effects on intracellular ROS generation. Coumaric acid and resveratrol promoted a rapid increase in intracellular ROS production and pro-oxidant effects, while the exposition of cultured ECs to caffeic acid resulted in a significant anti-oxidant effect (Fig. 2A). Chlorogenic acid, thought exerting an overall pro-oxidant outcome, displayed dual behavior on intracellular ROS generation showing both inhibiting and activating effects (Fig. 2A). Since the antioxidants were used at the same concentration, these results suggest that the current observed behavior may depend upon intrinsic chemical and structural characteristics of the tested compounds. Such a hypothesis is supported by the finding that caftaric acid and ferulic acid, 

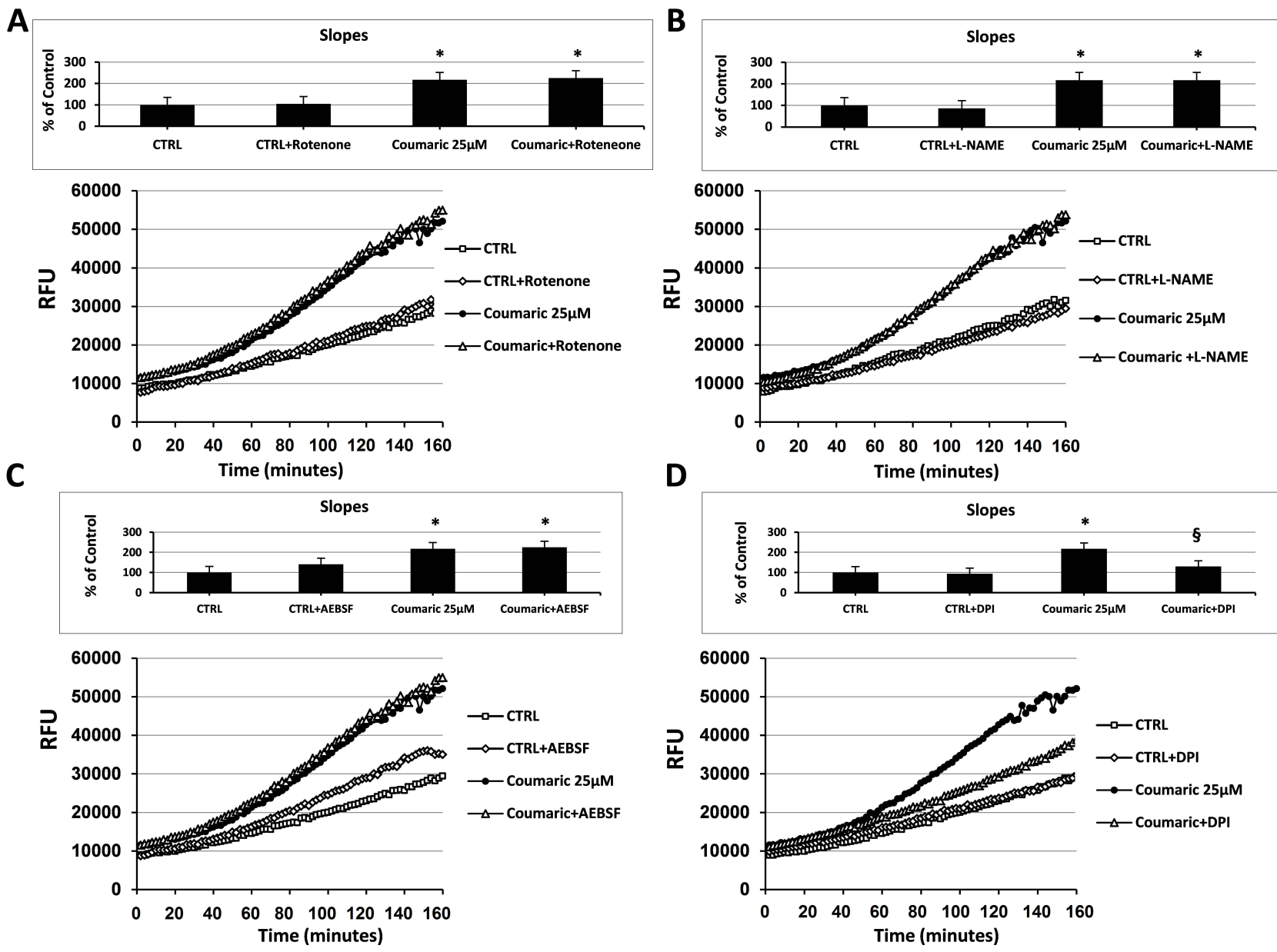

Fig. (5). Effect of different inhibitors of ROS-generating enzymes on coumaric acid-induced modification of the intracellular ROS levels. Measurement of intracellular ROS generation in cultured human ECs in the absence (CTRL all panels) or presence of (A-D) $25 \mu \mathrm{M}$ coumaric acid; (A) $10 \mu \mathrm{M}$ rotenone, or $25 \mu \mathrm{M}$ coumaric acid + $10 \mu \mathrm{M}$ rotenone; (B) $1 \mathrm{mM}$ L-NAME, or $25 \mu \mathrm{M}$ coumaric $+1 \mathrm{mM}$ L-NAME; (C) 10 $\mu \mathrm{M}$ AEBSF, or $25 \mu \mathrm{M}$ coumaric acid + $10 \mu \mathrm{M}$ AEBSF; (D) $20 \mu \mathrm{M}$ DPI, or $25 \mu \mathrm{M}$ coumaric acid + $20 \mu \mathrm{M}$ DPI. Treatment-induced variation of fluorescence was kinetically measured every minute for 120 consecutive minutes as described in materials and methods. (Figure insets): Using as reference untreated cells, the overall anti- and pro-oxidant effect was extrapolate by comparison of the slope obtained from at least five kinetic measurements for each experimental condition. Data are expressed as percentage of control and represent the means \pm SE of five different experiments. * Significantly different from CTRL at $\mathrm{P}<0.05$. $\S$, significantly different from $25 \mu \mathrm{M}$ coumaric acid at at $\mathrm{P}<0.05$

which are chemically and structurally related to chlorogenic acid and caffeic acid, respectively, evoked the same effect on intracellular ROS generation (Fig. 2B).

Even though in a different concentrations range, all the antioxidants displayed a concentration-dependent effect on intracellular ROS levels, which resulted in different anti-and pro-oxidant behavior. At low concentration all the antioxidants showed, or leaned toward an anti-oxidant effect, while the increase of concentration induced, or had a tendency to promote a pro-oxidant outcome. The present results clearly indicate a relationship between the dose applied and the potential ability of these molecules to act both as anti- and prooxidants. Similar behavior has been reported with others antioxidants in several experimental models [11, 12]. However since antioxidant behavior can vary between types of cells and can differ among molecules, the mechanisms involved in such phenomena are poorly understood and still under inves- tigation. Here we suggest flavin containing detoxifying endoplasmatic reticulum (ER)-resident enzymes as responsible for the observed antioxidant-induced rise in intracellular ROS level.

Major intracellular sources of ROS encompass mitochondria, NADPH oxidase, NOS and smooth ER. Smooth ER (lacking bound ribosomes) contains enzymes that catalyze a series of reactions to detoxify lipid-soluble drugs and other harmful metabolic products. The most extensively studied of these is the flavin-containing cytochrome $P-450$ (CYP) families of enzymes that can oxidize unsaturated fatty acids and xenobiotics and reduce molecular $\mathrm{O}_{2}$ to produce $\mathrm{O}_{2}$. and/or $\mathrm{H}_{2} \mathrm{O}_{2}$ [23-25]. Under our experimental conditions, the antioxidant-induced intracellular ROS generation is unlikely to be attributable to mitochondria, NOS or NADPH oxidases, since inhibitors of these ROS-generating systems failed to abolish such an effect. While involvement of ER- 

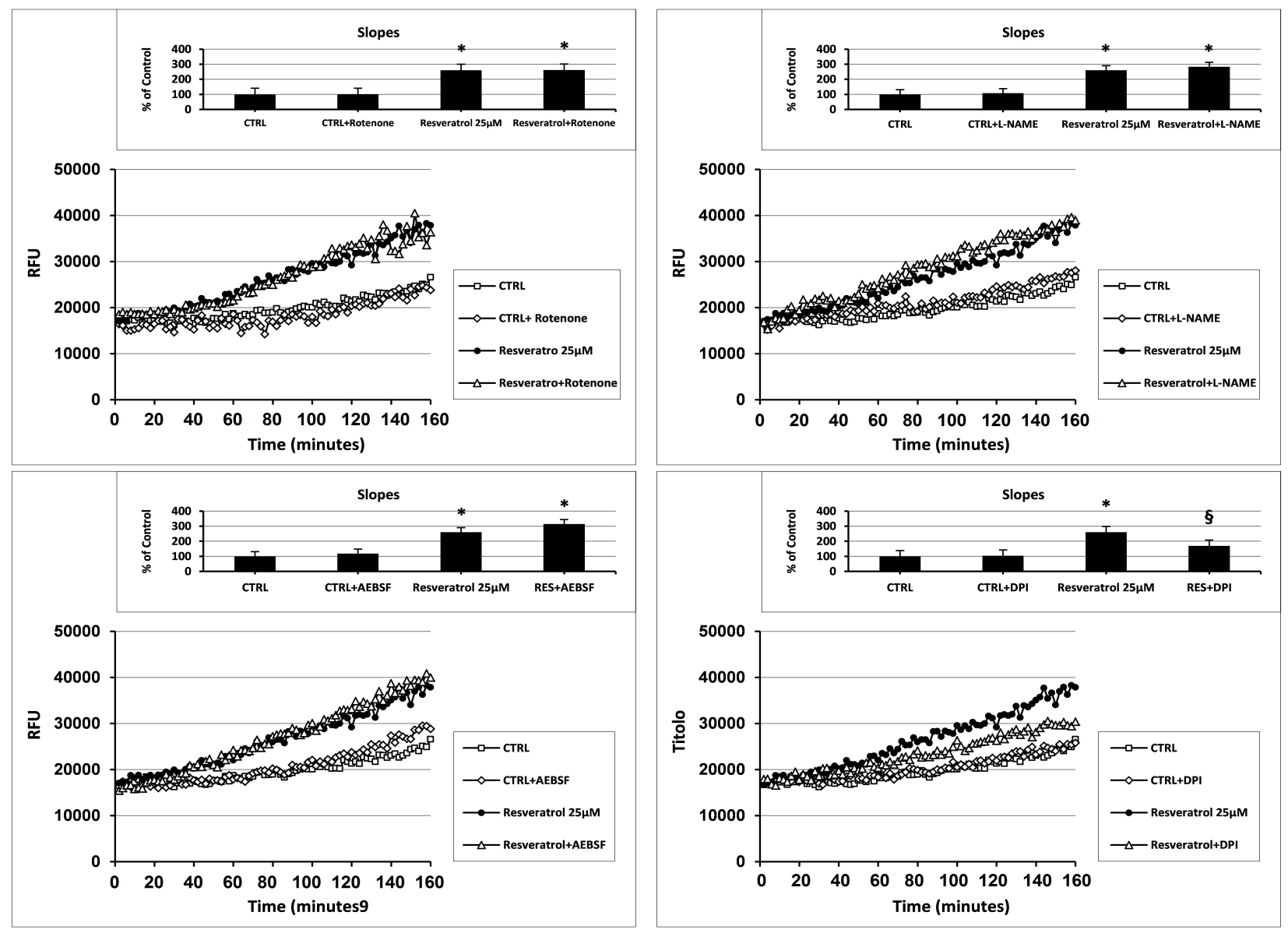

Fig. (6). Effect of different inhibitors of ROS-generating enzymes on Resvertarol-induced modification of the intracellular ROS levels. Measurement of intracellular ROS generation in cultured human ECs in the absence (CTRL all panels) or presence of (A-D) $25 \mu \mathrm{M}$ resveratrol; (A) $10 \mu \mathrm{M}$ rotenone, or $25 \mu \mathrm{M}$ resveratrol $+10 \mu \mathrm{M}$ rotenone; (B) $1 \mathrm{mM}$ L-NAME, or $25 \mu \mathrm{M}$ resveratrol + $1 \mathrm{mM} \mathrm{L}-\mathrm{NAME}$; (C) $10 \mu \mathrm{M}$ AEBSF, or $25 \mu \mathrm{M}$ resveratrol $+10 \mu \mathrm{M}$ AEBSF; (D) $20 \mu \mathrm{M}$ DPI, or $25 \mu \mathrm{M}$ resveratrol $+20 \mu \mathrm{M}$ DPI. Treatment-induced variation of fluorescence was kinetically measured every minute for 120 consecutive minutes as described in materials and methods. (Figure insets): Using as reference untreated cells, the overall anti- and pro-oxidant effect was extrapolate by comparison of the slope obtained from at least five kinetic measurements for each experimental condition. Data are expressed as percentage of control and represent the means \pm SE of five different experiments. * Significantly different from CTRL at $\mathrm{P}<0.05$. §, significantly different from $25 \mu \mathrm{M}$ resveratrol at $\mathrm{P}<0.05$.

resident CYP families in the antioxidant-induced pro-oxidant effect is suggested by the finding that the flavoprotein inhibitor DPI completely counteracted the raise in intracellular ROS elicited by the tested compounds. Such a hypothesis is strongly supported by several observations. I) DPI is a general flavoprotein inhibitor and members of the ER-resident CPY families are mainly flavin adenosine dinucleotide (FAD)- and flavin mononucleotide (FMN)-containing enzymes [23-25]. ii) The observed shift from anti-oxidant to pro-oxidant behavior always took place with increasing concentrations and this is consistent with the intervention of ERresident CYP enzymes, which may see antioxidants as xenobiotics when supplied to cells at high concentration iii) NADPH oxidases also containing FAD and NAD, however apocynin and AEBSF which inhibit NADPH oxidases with a different mechanism than DPI, did not produce any inhibitory effect on antioxidant-induced ROS generation.
In our hands, chlorogenic acid showed a dual anti- and pro-oxidant effect, in relation to different intracellular ROS levels. Indeed, regardless of the initial ROS levels, coumaric acid and resveratrol always acted as pro-oxidants and caffeic acid as an anti-oxidant, while the treatment of cultured ECs with chlorogenic acid resulted in a double effect which was pro-oxidant at low initial ROS level and anti-oxidant at high (Fig. 3). It may be possible that ECs with a low oxidative state recognize chlorogenic acid like a dangerous compound rather than an antioxidant and remove it through xenobiotic metabolism. On the other hand when the cellular oxidative state is high, chlorogenic acid may be normally seen by the ECs as antioxidant and used in order to counteracts high intracellular ROS leves and promote a more optimal oxidative state. In this regard, a similar dual-behavior has been reported for other antioxidants, both in vitro and in vivo experimental models $[13,14]$. The different ROS levels observed at the beginning of the experiments could be related 
to the use of a heterogeneous cell population obtained from human umbilical cords of women born in Sardinia, where G6PD deficiency is very frequent [26]. As ECs used in the current work derived from the umbilical cord of Sardinian women, and since G6PD deficiency promotes endothelial oxidant stress by aberrantly regulating intracellular ROS level [27], such a hypothesis may be conceivable.

The current results demonstrate intracellular oxidative effect of NA due to high-dose administration in cultured ECs and may pave the way for understanding the mechanisms involved in many of their reported untoward effect. Epidemiological studies demonstrate a significant inverse correlation between polyphenol-rich foods consumption and cardiovascular risk [28]. Nonetheless, many human intervention trials, some meta-analyses and other studies indicate that antioxidants not only fail to protect against CVD [22], but also that some of them may accelerate development vascularrelated pathologies in certain subjects [29]. Explanation for these results is still unclear. Some authors suggest that dietary polyphenols may be typical xenobiotics, metabolized as such by the cell and rapidly degraded to produce toxic byproducts including ROS [30]. Thus, they may be beneficial in the correct amounts but high concentration of these compounds may not be good. Furthermore, oxidant and antioxidant status of both patients and people should be probably checked before undergoing high antioxidant intake or supplementation [31, 32]. Indeed, despite diffuse present contradictions, it appears that at least populations with insufficient or unbalanced nutritional levels may benefit from an increased intake of dietary antioxidants or supplements [33]. It may be possible that people with an altered individual oxidative state, for example people with a G6PD deficiency, may have beneficial improvement from an antioxidantenriched diet, whereas this may be dangerous for healthy people with a normal individual oxidative state. Intracellular ROS modification is a potential double-edged sword in disease prevention and promotion. While generation of ROS was once viewed as the major cause of pathological conditions, compelling evidences are now indicating that ROS play crucial roles in normal physiological processes. In particular it appears that precise regulation of intracellular ROS levels are needed to allow normal cell function [34], thus antioxidant-based strategies aimed at inhibiting the production of intracellular ROS may have the unpleasant effect of promoting EC death.

Due to the widely promoted, tantalizing union of antioxidants and good health, enriched antioxidant diets and/or antioxidant supplementation has become a lifestyle. Our results indicate that food-derived phenolic compounds may not only counteract the negative effect of oxidative stress but could also promote EC damage by causing an imbalance of the intracellular ROS levels. Since there is a substantial body of published work that shows NA can reach in vivo concentrations comparable to the ones we used in vitro, we suggest our results could be representative of a physiologically relevant in vivo mechanism.

\section{CONFLICT OF INTEREST}

The authors confirm that this article content has no conflicts of interest.

\section{ACKNOWLEDGEMENTS}

Work funded by the Italian Ministry of University (PRIN 08), Banco di Sardegna Foundation and Sardinia Region L.R. n7 2007.

\section{SUPPLEMENTARY MATERIAL}

Supplementary material is available on the publisher's web site along with the published article.

\section{REFERENCES}

[1] Bergamini, C.M.; Gambetti, S.; Dondi, A. Cervellati, C. Oxygen, reactive oxygen species and tissue damage. Curr. Pharm. Des., 2004, 10, 1611-1626.

[2] Stoclet, J.C.; Chataigneau, T.; Ndiaye, M.; Oak, M.H.; El Bedoui, J.; Chataigneau, M. Schini-Kerth, V.B. Vascular protection by dietary polyphenols. Eur. J. Pharmacol., 2004, 500, 299-313.

[3] Carluccio, M.A.; Siculella, L.; Ancora, M.A.; Massaro, M.; Scoditti, E.; Storelli, C.; Visioli, F.; Distante, A. De Caterina, R. Olive oil and red wine antioxidant polyphenols inhibit endothelial activation - Antiatherogenic properties of Mediterranean diet phytochemicals. Arterioscler. Thromb. Vasc. Biol., 2003, 23, 622-629.

[4] Rah, D.K.; Han, D.W.; Baek, H.S.; Hyon, S.H.; Park, J.C. Prevention of reactive oxygen species-induced oxidative stress in human microvascular endothelial cells by green tea polyphenol. Toxicol. Lett., 2005, 155, 269-275.

[5] Kris-Etherton, P.M.; Keen, C.L. Evidence that the antioxidant flavonoids in tea and cocoa are beneficial for cardiovascular health. Curr. Opin. Lipidol., 2002, 13, 41-49.

[6] Dimmeler, S.; Zeiher, A.M. Reactive oxygen species and vascular cell apoptosis in response to angiotensin II and pro-atherosclerotic factors. Regul. Pept., 2000, 90, 19-25.

[7] Irani, K. Oxidant signaling in vascular cell growth, death, and survival : a review of the roles of reactive oxygen species in smooth muscle and endothelial cell mitogenic and apoptotic signaling. Circ. Res., 2000, 87, 179-183.

[8] Spitaler, M.M.; Graier, W.F. Vascular targets of redox signalling in diabetes mellitus. Diabetologia., 2002, 45, 476-494.

[9] Willcox, B.J.; Curb, J.D.; Rodriguez, B.L. Antioxidants in cardiovascular health and disease: key lessons from epidemiologic studies. Am. J. Cardiol., 2008, 101, 75D-86D.

[10] Maulik, N.; Das, D.K. Redox signaling in vascular angiogenesis. Free. Radic. Biol. Med., 2002, 33, 1047-1060.

[11] Galati, G.; Sabzevari, O.; Wilson, J.X.; O'Brien, P.J. Prooxidant activity and cellular effects of the phenoxyl radicals of dietary flavonoids and other polyphenolics. Toxicology, 2002, 177, 91-104.

[12] Pasciu, V.; Posadino, A.M.; Cossu, A.; Sanna, B.; Tadolini, B.; Gaspa, L.; Marchisio, A.; Dessole, S.; Capobianco, G.; Pintus, G. Akt downregulation by flavin oxidase-induced ROS generation mediates dose-dependent endothelial cell damage elicited by natural antioxidants. Toxicol. Sci., 2010, 114, 101-112.

[13] Gadacha, W.; Ben-Attia, M.; Bonnefont-Rousselot, D.; Aouani, E.; Ghanem-Boughanmi, N.; Touitou, Y. Resveratrol opposite effects on rat tissue lipoperoxidation: pro-oxidant during day-time and antioxidant at night. Redox. Rep., 2009, 14, 154-158.

[14] Carru, C.; Pasciu, V.; Sotgia, S.; Zinellu, A.; Nicoli, M.C.; Deiana, L.; Tadolini, B.; Sanna, B.; Masala, B.; Pintus, G. The Oxidative State of LDL is the Major Determinant of Anti/Prooxidant effect of coffee on Cu catalysed peroxidation. Open. Biochem. J., 2011, 5, 18.

[15] Zinellu, A.; Sotgia, S.; Scanu, B.; Pintus, G.; Posadino, A.M.; Cossu, A.; Deiana, L.; Sengupta, S.; Carru, C. S-homocysteinylated LDL apolipoprotein B adversely affects human endothelial cells in vitro. Atherosclerosis, 2009, 206, 40-46. 
[16] Pintus, G.; Tadolini, B.; Posadino, A.M.; Sanna, B.; Debidda, M.; Carru, C.; Deiana, L.; Ventura, C. PKC/Raf/MEK/ERK signaling pathway modulates native-LDL-induced E2F-1 gene expression and endothelial cell proliferation. Cardiovasc. Res., 2003, 59, 934944.

[17] Yuhki, K.; Miyauchi, T.; Kakinuma, Y.; Murakoshi, N.; Maeda, S.; Goto, K.; Yamaguchi, I.; Suzuki, T. Endothelin-1 Production Is Enhanced by Rotenone, a Mitochondrial Complex I Inhibitor, in Cultured Rat Cardiomyocytes. J. Cardiovasc. Pharmacol., 2001, 38, 850 .

[18] Therade-Matharan, S.; Laemmel, E.; Duranteau, J.; Vicaut, E. Reoxygenation after hypoxia and glucose depletion causes reactive oxygen species production by mitochondria in HUVEC. Am. J. Physiol. Regul. Integr. Comp. Physiol., 2004, 287, R1037-R1043.

[19] San Martin, A.; Foncea, R.; Laurindo, F.; Ebensperger, R.; Griendling, K.; Leighton, F. Nox1-based NADPH oxidase-derived superoxide is required for VSMC activation by advanced glycation endproducts. Free Radic. Biol. Med., 2007, 42, 1671-1679.

[20] Davidge, S.T.; Baker, P.N.; Laughlin, M.K.; Roberts, J.M. Nitric oxide produced by endothelial cells increases production of eicosanoids through activation of prostaglandin $\mathrm{H}$ synthase. Circ. Res., 1995, 77, 274-283.

[21] Posadino, A.M.; Porcu, M.C.; Marongiu, B.; Cossu, A.; Piras, A.; Porcedda, S.; Falconieri, D.; Cappuccinelli, R.; Biosa, G.; Pintus, G.; Pretti, L. Antioxidant activity of supercritical carbon dioxide extracts of Salvia desoleana on two human endothelial cell models. Food Res. Int., 2012, 46, 354-359.

[22] Willcox, B.J.; Curb, J.D.; Rodriguez, B.L. Antioxidants in cardiovascular health and disease: Key lessons from epidemiologic studies. Am. J. Cardiol., 2008, 101, 75d-86d.

[23] Guengerich, F.P. Cytochrome P450 and chemical toxicology. Chem. Res. Toxicol., 2008, 21, 70-83.
[24] Puntarulo, S.; Cederbaum, A.I. Production of reactive oxygen species by microsomes enriched in specific human cytochrome P450 enzymes. Free. Radic. Biol. Med., 1998, 24, 1324-1330.

[25] Neve, E.P.A. Ingelman-Sundberg, M. Cytochrome P450 proteins: Retention and distribution from the endoplasmic reticulum. Curr. Opin. Drug. Discov. Dev., 2010, 13, 78-85.

[26] De Vita, G.; Alcalay, M.; Sampietro, M.; Cappelini, M.D.; Fiorelli, G.; Toniolo, D. Two point mutations are responsible for G6PD polymorphism in Sardinia. Am. J. Hum. Genet., 1989, 44, 233-240.

[27] Leopold, J.A.; Cap, A.; Scribner, A.W.; Stanton, R.C.; Loscalzo, J. Glucose-6-phosphate dehydrogenase deficiency promotes endothelial oxidant stress and decreases endothelial nitric oxide bioavailability. FASEB J., 2001, 15, 1771-1773.

[28] Kaliora, A.C.; Dedoussis, G.V.; Schmidt, H. Dietary antioxidants in preventing atherogenesis. Atherosclerosis, 2006, 187, 1-17.

[29] Lee, D.H.; Folsom, A.R.; Harnack, L.; Halliwell, B.; Jacobs, D.R. Does supplemental vitamin $C$ increase cardiovascular disease risk in women with diabetes? Am. J. Clin. Nutr., 2004, 80, 1194-1200.

[30] Halliwell, B. Polyphenols: antioxidant treats for healthy living or covert toxins? J. Sci. Food. Agric., 2006, 86, 1992-1995.

[31] Steinberg, D.; Witztum, J. Is the oxidative modification hypothesis relevant to human atherosclerosis? Do the antioxidant trials conducted to date refute the hypothesis? Circulation, 2002, 105, 21072111.

[32] Violi, F.; Micheletta, F.; Iuliano, L.; Steinberg, D.; Witztum, J. How to select patient candidates for antioxidant treatment?* Response. Circulation, 2002, 106, e195.

[33] Seifried, H.; Anderson, D.; Fisher, E.; Milner, J. A review of the interaction among dietary antioxidants and reactive oxygen species. J. Nutr. Biochem., 2007, 18, 567-579.

[34] Martindale, J.L.; Holbrook, N.J. Cellular response to oxidative stress: signaling for suicide and survival. J. Cell. Physiol., 2002, 192, 1-15. 